Contents List available at VOLKSON PRESS

Intelligent Computing and Information Engineering (ICIE )

DOI : http://doi.org/10.26480/icie.01.2017.105.107

Journal Homepage: : https://www.intelcomp-design.com/

\title{
RESEARCH ON THE A CLASS MAINTENANCE SCHEDULING IN THERMAL POWER GENERATING UNITS BASED ON THE CRITICAL CHAIN PROJECT MANAGEMENT
}

\author{
Jing-Min Wang, Hang Zhang* \\ North China Electric Power University, Hebei, Baoding, 071003, China
}

*Corresponding author email: dianqijiaojian_123@163.com

This is an open access article distributed under the Creative Commons Attribution License, which permits unrestricted use, distribution, and reproduction in any medium, provided the original work is properly cited

\section{ARTICLE DETAILS}

\section{Article History:}

Received 12 May2017

Accepted 12 July 2017

Available online 14 September 2017

Keywords:

Critical Chain Project

Management, A Class

Maintenance Project, Time

\section{ABSTRACT}

In order to shorten the duration in the A class maintenance project of the thermal power generating unit, the critical chain project management is introduced. On the basis of ensuring the accuracy and simplicity of the calculation, the method of estimating the duration for the project is determined. The research has important reference value for the future A class maintenance project of the power plant.

\section{INTRODUCTION}

China issued the Guidance on Equipment Maintenance of Power Generation Enterprises (DL / T 838-2003) which divides maintenance into A, B, C, D four levels. A Class Maintenance refers to the generator set to conduct a comprehensive disintegration inspection and repair, to maintain, restore or improve equipment performance. This means that the unit has to stop operating, and takes a long time. However, the unit for a long time outage will affect not only the power plant's own economic benefits, but also the power system, and even social life. Therefore, in the shortest possible time to complete the maintenance of high quality is particularly important.

In the current A class maintenance, there is a common problem that maintenance duration is too long. In order to ensure that the maintenance project is completed on time, duration is estimated by low risk, which led to the project safety time is too long, or even up to $200 \%$.

In this paper, the Critical Chain Project Management is introduced into the A class maintenance project of the thermal power generating unit. The methods to solve the problem are put forward according to the characteristics and actual situation.

\section{CRITICAL CHAIN PROJECT MANAGEMENT}

In 1997, Dr. Goldratt used the method of Theory of Constraints to analyze the progress of the project, and put forward a new scheduling method Critical Chain Project Management (CCPM) [1]. The CCPM is the longest task chain that satisfies the resource constraints and interdependence of activities [2]. CCPM estimates project duration non-conservatively, weakened the psychological factors on the impact of the duration, to achieve shortening the duration.

In general, the relationship between the probability of activity completion and the time is shown in Figure 1. A is the median of the probability, that is, the probability of completing is $50 \%$. Uncertainty factors lead to the back longer, the higher the uncertainty, the longer [3].

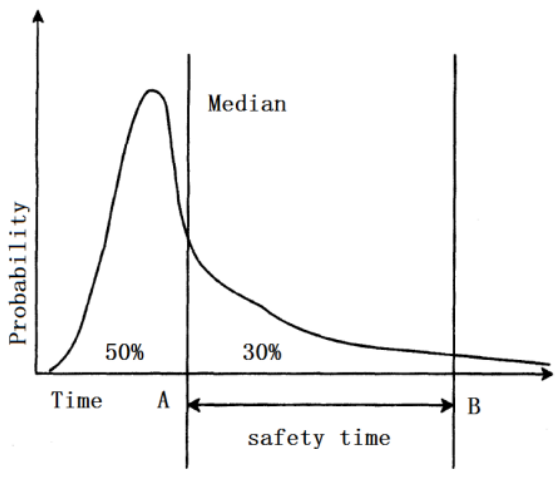

Figure 1: Relationship between probability and time 
CCPM considers that the difference between A and B for the safety time, to mitigate the effects of uncertainty. The normal working time at $50 \%$ of the completion probability; and $80 \%$ indicates the completion time for the working time of 2 times, $90 \%$ that the completion time for working time 3 times. Generally in the time estimation, the time will be set in the completion of the probability of $80 \%$ to $90 \%$, that is, B straight line shown. Thus, the general estimated time is 1 to 2 times more than working time.

\section{TIME ESTIMATION}

In order to improve the accuracy of the time estimation, and take into account the convenience of the actual estimation, this paper divides the time estimation method into with experience of maintenance and with no experience of maintenance.

\subsection{Time Estimation with No Experience of Maintenance}

For the power plants that have not undergone A class maintenance, this paper will use Gray Theory to estimate the working time [4]. A researcher mentioned that Gray is the concept between the White and Black. White refers to the system that the information is explicit and the data is complete. Black refers to the system that the information is implicit and the data is incomplete. Corresponding to Black and White, Gray refers to the system that part of the information is explicit and part of the data is complete [5-7]. A power plant that has not undergone A class maintenance fit the situation, so the Gray System Theory is introduced into the working time estimation [8].

Because it is the first estimate, the data is less, so to whiten them by the whitening weight function. Assuming that the segmentation interval $\Lambda=(\mathrm{m}, \mathrm{n}, \mathrm{p}$, q) of the whitening weight function $F_{\Lambda}(t)$ is the estimated duration of each activity of the project, the function is shown in Figure 2:

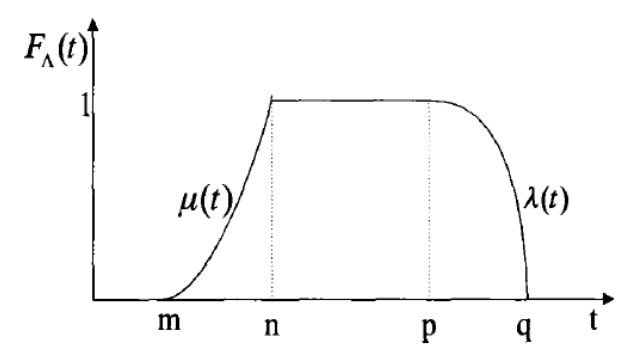

Figure 2: Whitening Weight Function

The horizontal axis represents the completion time, denoted by $t$. The vertical axis represents the probability or membership of the estimated duration, denoted by $F_{\Lambda}(t)$. The minimum time required for the activity to complete is denoted by $\mathrm{m}$, and the maximum time required for the activity to complete is denoted by q, and the minimum time required for the most likely execution of the activity is denoted by $\mathrm{n}$, and the maximum time required for the most likely execution of the activity to complete is denoted by $\mathrm{p}$, then $\mathrm{t}$ is determined by these four values. The function $F_{\Lambda}(t)$ is the third-order function. In the interval $[\mathrm{m}, \mathrm{n})$, the function follows $\mu(\mathrm{t})$ and it is an exponential increasing function. In the interval $(\mathrm{p}, \mathrm{q}]$, the function follows $\lambda(\mathrm{t})$ and it is a non - linear function of increasing slope. [n, $\mathrm{p}]$ is the most likely interval in which the probability or membership degree is 1 . According to the graph, $F_{\Lambda}(t)$ is:

$F_{\Lambda}(t)=\left\{\begin{array}{c}\mu(\mathrm{t}), \mathrm{t} \in[\mathrm{m}, \mathrm{n}) \\ 1, \mathrm{t} \in[\mathrm{n}, \mathrm{p}] \\ \lambda(\mathrm{t}), \mathrm{t} \in(\mathrm{p}, \mathrm{q}]\end{array}\right.$

This function is theoretically feasible, but the time estimation does not need to be so precise actually. In the principle of simple and easy to operate, in this paper, the function is deformed, and $\mu(t), \lambda(t)$ are simplified to linear function, which is shown in Figure 2-2:

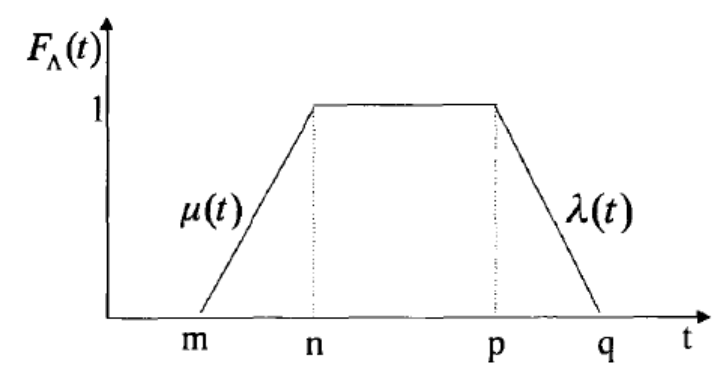

Figure 3: Deformed Whitening Weight Function

After deformation, the $F_{\Lambda}(t)$ function is:

$$
F_{\Lambda}(t)=\left\{\begin{array}{c}
\mu(\mathrm{t})=\frac{t-m}{n-m}, \mathrm{t} \in[\mathrm{m}, \mathrm{n}) \\
1, \mathrm{t} \in[\mathrm{n}, \mathrm{p}] \\
\lambda(\mathrm{t})=\frac{q-t}{q-p}, \mathrm{t} \in(\mathrm{p}, \mathrm{q}]
\end{array}\right.
$$

The expected duration Exp (i) for each activity i $(i=1,2 \cdot \bullet n)$ is:

$\operatorname{Exp}(\mathrm{i})=\frac{\int_{m}^{q} F_{\Lambda}(t) t d t}{\int_{m}^{q} F_{\Lambda}(t) d t}$

According to this formula, when the four durations of $\mathrm{m}, \mathrm{n}, \mathrm{p}, \mathrm{q}$ are predicted and the whitening weight function $F_{\Lambda}(t)$ is unknown, the expected working time is organized as follows:

$$
\operatorname{Exp}(\mathrm{i})=\frac{2 m_{i}+n_{i}+p_{i}+q_{i}}{6}
$$


When estimating these four durations, we should forecast q firstly. As mentioned at former, according to theoretical and practical experience, the estimated time is generally $80 \%$ to $90 \%$ of the completion probability, based on the estimated time plus the right time, which is to complete the activities of the longest time $\mathrm{q}$. And time $\mathrm{n}$ and $\mathrm{p}$ is closer to half of the value $\mathrm{q}$, so, as long as addition and subtraction of the appropriate time on the basis of $50 \%$ of the value $\mathrm{q}$, that is $\mathrm{n}$ and $\mathrm{p}$. The value $\mathrm{m}$ is the minimum time required to complete the activity, which is an ideal state and cannot be achieved in practice. Therefore, when estimating the value, actual factors do not to be considered, assuming sufficient resources, workers skilled, risk-free.

\subsection{Time Estimation with Experience of Maintenance}

Dr. Goldratt proposed that the actual working time of the activity is half of the historical duration. This method is the easiest to estimate the actual working time in the case of historical data.

Later, the probability method is proposed, which calculates the actual working time is based on the probability. Assuming that working time of a project is usually estimated at $80 \%$ of the completion probability, that is 2 times of the working time, so the working time of the activity is $\frac{1}{2}$ of the historical duration of every activity. Similarly, according to $90 \%$ of the probability of completion to estimate, the working time of the activity is $\frac{1}{3}$ of the historical duration of every activity. The method is simple and accurate, and meets the requirements of the actual project.

\section{CONCLUSIONS}

In this paper, the CCPM is introduced into the A class maintenance scheduling of thermal power generating units to solve the common problem. In order to solve the problem that duration estimation is too long and ensure the accuracy and simplicity of the estimation, the estimated duration of the project is divided into two cases. For the project with no maintenance experience, the gray theory is suggested to use; for the project with maintenance experience, the probability method is suggested to use.

\section{REFERENCES}

Goldratt, E.M. 1997. Critical chain. The North River Press.

Jing-wen, Z., Ruo-nan, L. 2013. Review of critical chain project scheduling method. Control and Decision, 28 (9),1281-1287.

[3] Fu-zhou, L., Jing, L. 2013. Multi-objective comprehensive optimization research on critical buffer in construction project. Journal of Xi'an University of Architecture and Technology (Natural Science Edition), (06), 902-906

[4] Shan-cong, Z., Li-cong, M. 2016. Discussion on Critical Chain Risk Buffer of High tech Project Fuzzy Evaluation Method Based on Entropy. Modern Management Science, (10), 9-11.

[5] Rahman, S. 1998. Theory of constraints: A review of the philosophy and its applications. International Journal of Operations abd Production Management, 18 (4), 336-355.

[6] Li, B. 2012. Study on Methods of Buffer Estimating and Monitoring in Critical Chain Project Management. Huazhong University of Science and Technology.

[7] Leach, L.P. 2000. Critical Chain Project Management. The Wiley Guide to Managing Projects, 35.

Yu, Z. 2009. Research of Project Process Management Based on Critical Chain. Dalian University of Technology. 